\title{
FOREWORD
}

\section{THE FIRST TEN YEARS AND THE FUTURE HORIZON}

\author{
THOMAS R. ODHIAMBO \\ The International Centre of Insect Physiology and Ecology (ICIPE), P.O. Box 30772, Nairobi, Kenya
}

Ten years is a long time for a journal in any scientific field in any developing region of the world. The rate of demise for such journals is a recurring statistic familiar to most scientists in the developing world as well as those who support or emphathise with the scientific community in the developing world. It was therefore not surprising that, when the founding of Insect Science and Its Application was mooted a dozen years ago, the idea was met with coolness and scepticism.

As we celebrate the tenth anniversary of this international bimonthly journal of tropical insect science, we do so with confidence for its future growth in stature and essence, based on a solid achievement which has been incremental in quality and content each succeeding year from its inception. All the four targets we set ourselves at the very beginning of this voyage in international scientific publishing have been reached within this first decade:

(1) We have set a very high quality standard for the editorial content of the journal, based on an extensive peer review protocol and a functioning international board.

(2) We have maintained a wide thematic diversity of the disciplines encompassed within the journal- from natural history to endocrinology, from bionomics studies to population modelling, from pest control methodologies to evolutionary theories.

(3) We have increasingly widened our sources of journal content from tropical and subtropical Africa to other tropical and subtropical regions of the world.
(4) We have succeeded in having all processes of publishing (editorial and peer review, design and formating, printing and binding, distribution and circulation, etc.) centred within an international communication centre located in a developing country.

We believe that all these accomplishments augur well for the second decade of the journal's life.

The primary purpose of Insect Science and Its Application is to provide an interactive pan-tropical forum for the dissemination of the new advances in tropical insect science in all its multi-disciplinary range, and for the review and discussion of these advances in terms of the evolution of this scientific area as well as its application to human welfare in its broadest sense. This is a vitally important task, and one which demands a continuing concern for excellence and relevance. In this connection, as we did 2 years ago, we will carry out a thorough-going review of the journal in 1992: its content, its quality, and whether it meets the readership's needs.

We very much hope that our authors, readers and supporters alike will celebrate this tenth year by making Volume 10 of Insect Science and Its Application a memorable one by its content of pithy reviews, giving a wide sweep of research developments over the last 10 years, while providing an incisive critique of these advances within a tropical ecological context. 
As we close the life of the ten volumes of the journal, we wish to be reminded of the prospects of the journal by some of our contributors and readers:

Insect Science and Its Application has considerably improved and is now a full international scientific review. I had the proof of its international audience when I published a mini-review on Agriculture and Vector Resistance in the journal. More than 100 reprints were requested to me, which is very satisfactory.
The journal continues to progress under your able leadership as Editor-in-Chief. We appreciate your efforts on behalf of this excellent joumal.

Fowden G. Maxwell Texas A \& M University College Station, Texas, USA

The journal prestige is high and I believe that it will keep growing.

$$
\begin{array}{r}
\text { Vladimir Landa } \\
\text { Czechoslovak Academy of Sciences } \\
\text { Czechoslovakia }
\end{array}
$$

J. Mouchet

ORSTOM

Paris, France
I am indeed pleased that you and your board continue to solicit and publish research papers with worldwide application.

R. A. Brust

The University of Manitoba Winnipeg, Manitoba, Canada 